\title{
Analysis of the process of representing clinical statements for decision-support applications: a comparison of openEHR archetypes and HL7 virtual medical record
}

\author{
A. González-Ferrer ${ }^{1}$, M. Peleg ${ }^{2}$, M. Marcos ${ }^{3}$, J.A. Maldonado ${ }^{4,5}$ \\ ${ }^{I}$ Fundación de Investigación Biomédica, Hospital Clínico San Carlos; Instituto de \\ Investigación Sanitaria San Carlos (IdISSC), 28040 Madrid, Spain \\ ${ }^{2}$ Department of Information Systems, University of Haifa, Haifa, Israel, 3498838 \\ ${ }^{3}$ Department of Computer Engineering and Science, Universitat Jaume I, Av. de \\ Vicent Sos Baynat s/n, 12071 Castellón, Spain \\ ${ }^{4}$ Instituto de Aplicaciones de las Tecnologías de la Información y de las \\ Comunicaciones Avanzadas, Universitat Politècnica de València, Valencia, Spain \\ ${ }^{5}$ VeraTech for Health SL, Valencia, Spain
}

Corresponding Author:

Arturo González-Ferrer

Fundación de Investigación Biomédica, Hospital Clínico San Carlos

C/ Prof. Martín Lagos, S/N 28040, Madrid, Spain

E-mail: arturogf@gmail.com

Phone number: +34913303735

The final publication is available at Springer via http://dx.doi.org/10.1007/s10916-016-0524-3 


\begin{abstract}
Objectives. Delivering patient-specific decision-support based on computer-interpretable guidelines (CIGs) requires mapping CIG clinical statements (data items, clinical recommendations) into patients' data. This is most effectively done via intermediate data schemas, which enable querying the data according to the semantics of a shared standard intermediate schema. This study aims to evaluate the use of HL7 virtual medical record (vMR) and openEHR archetypes as intermediate schemas for capturing clinical statements from CIGs that are mappable to electronic health records (EHRs) containing patient data and patient-specific recommendations. Methods. Using qualitative research methods, we analyzed the encoding of ten representative clinical statements taken from two CIGs used in real decision-support systems into two health information models (openEHR archetypes and HL7 vMR instances) by four experienced informaticians.

Results. Discussion among the modelers about each case study example greatly increased our understanding of the capabilities of these standards, which we share in this educational paper. Differing in content and structure, the openEHR archetypes were found to contain a greater level of representational detail and structure while the vMR representations took fewer steps to complete.

Conclusions. The use of openEHR in the encoding of CIG clinical statements could potentially facilitate applications other than decision-support, including intelligent data analysis and integration of additional properties of data items from existing EHRs. On the other hand, due to their smaller size and fewer details, the use of vMR potentially supports quicker mapping of EHR data into clinical statements.
\end{abstract}

Keywords: clinical decision-support systems, clinical guidelines, computerinterpretable guidelines, data integration, openEHR archetypes, $H L 7$ virtual Medical Record (vMR)

\title{
Introduction
}

Computer-interpretable guidelines (CIGs)[1] are promising tools for delivering patient-specific decision support effectively. Their implementation requires mapping CIG knowledge to patient data. The knowledge consists of clinical statements used in CIG tasks (e.g., decisions, actions) that refer to clinical concepts (e.g., blood pressure). In ubiquitous decision-support systems (DSS)[2], EHRs go beyond the core EHR[3] including also sensor-acquired data, patients' 
self-reported data, and DSS-generated recommendations and abstractions. The medical concept's level of abstraction, and the terminology used by the CIG and its data sources often differ, hence a mapping between the global CIG schema and the local schemas should be established[4]. This allows the CIG to be executed for a specific patient. To aid in the mapping task, using an intermediate (mediated) schema providing a reconciled, integrated and virtual view of the local repositories is a feasible solution $[5,6]$. The mediated schema is designed to represent the attributes of the domain relevant to the integration application and does not necessarily represent all of the attributes available in the sources[7]. When a CIG is executed and a clinical statement needs to be evaluated with concrete patient data, the data query is posed in terms of the mediated schema, rather than the individual local schemas.

In this study, we considered two EHR information models as mediated schemas, namely HL7' virtual medical record (vMR) R1 and openEHR archetypes. We used these standards to represent clinical statements of CIGs that are mappable to EHRs. We then compared the representation process and the resulting artifacts using case-study examples and structured meetings among experts. We report our results and conclusions about the suitability and uniqueness of these standards when used as intermediate schemas.

\section{Related Work}

There is a mature body of standards and open specifications covering the three layers of artifacts needed to represent the meaning of health data:

- Generic Reference Models (RM) containing a basic and stable framework for representing EHR components, that can be aggregated to create more complex components, and the context information necessary to meet ethico-legal requirements. Examples are ISO/EN13606-1, HL7 RIM, HL7 Clinical Document Architecture (CDA) standards or the openEHR RM.

- Clinical information models, which are detailed, reusable and domainspecific definitions of a clinical concept. Examples of such models are 
openEHR/ISO13606 archetypes, CDA templates, Detailed Clinical Models[8] and Clinical Element Models[9].

- Clinical terminologies are structured lists of terms which together with their definitions serve to describe unambiguously different aspects of healthcare. They have evolved from simple code-name-hierarchy arrangements, into rich, knowledge-based ontologies of medical concepts.

Several initiatives based their intermediate schema on a standard RM. The use of a simplified version of the HL7 RIM was selected by KDOM[5], MEIDA[10], and Cho et al.'s work [11]. A standard RM is necessary but may not be sufficient to constrain and represent specific semantics needed for DSS applications. For example, a standard RM may offer multiple ways of expressing the same clinical statements often required in DSS, such as adverse event. On the other hand, generic models contain many properties that are seldom used in DSSs. Therefore, specific clinical information models, where the assumptions about the representation of data are made explicit, provide a means for easily specifying more semantics than those provided by RMs. SAGE $[8,12]$ was the first to consider this requirement. It proposed the use of a virtual medical record, a simplified set of classes that a DSS would need to read and write data to an electronic patient record. Later, Detailed Clinical Models (DCM)[8] were created as a way to constrain aspects of those classes to specific clinical concepts. The former approach has been standardized by HL7 vMR standard [13] and the latter is similar to CEN/ISO EN13606 and openEHR archetypes. Marcos and Maldonado[6, 14] developed a comprehensive approach to deal with DSSs and EHRs interoperability based on archetypes. González-Ferrer and Peleg[15, 16] compared vMR, CDA and openEHR by evaluating their support of functional and nonfunctional requirements for interoperability. However, that study was limited since no experts in openEHR were involved and there was no analysis of the modeling process and the resulting representations. This work aims to include those aspects and to share our lessons learned. 


\section{Methods}

Four knowledge engineers represented the clinical statements of ten case-study examples in either openEHR or vMR, each using the standard which $\mathrm{s} / \mathrm{he}$ was familiar with (coauthors MP and AGF used vMR and MM together with JAM used openEHR). The convenience-based approach of familiarity of the model was used to establish correctness of the model; using non-expert modelers might result in incorrect models from which incorrect conclusions could be drawn. Notice that the usability of the tools was deliberately left outside the scope of this paper. The clinical statements (described in Table 1) constitute a representative sample (Appendix A) selected from a total of 117 statements from two CIGs used in the MobiGuide DSS project[2] and are based on clinical guidelines for managing atrial fibrillation[17] and gestational diabetes[18].

We used qualitative research methods to identify characteristic activities in the representation of CIG statements in openEHR and $\mathrm{vMR}$ and capabilities related to each of these standards. CIG statements were provided as textual descriptions (see Table 1). Both in the case of openEHR and $\mathrm{vMR}$, during a preparation phase, modelers analyzed the clinical statements and searched medical terminologies (UMLS [19] and SNOMED CT [20]) for relevant clinical terms. The utility of this phase is twofold: to gather ideas on the representation structures which fit best the statement, and to obtain terminological bindings that can be used to enrich these structures.

The next phase was the creation of instances corresponding to the clinical statements. For this purpose, vMR modelers directly defined the instances using a commercial XML editor together with the vMR schema, while the openEHR modeler used the LinkEHR archetype editor[21], including its mapping functionalities, to specialize the selected archetypes and generate instances based on them. The generated representations (artifacts, according to design science methodology[22]) were saved as XML files, that were made accessible to the four authors through a cloud storage service.

Our methodology focused on the artifacts produced. We carried out seven 2-hours structured meetings as consensus method[23], conducting artifact reviews in which the four experts discussed in detail all of the case study models, comparing the different solutions for each case and achieving consensus on observations. 


\section{Results}

Table 1 lists the clinical statements used in our analysis. Table 2 characterizes the statements in terms of the statement's category (general UMLS concept), its source and target, and a selection of SNOMED concepts that were deemed relevant for the statements. As can be observed, the statements cover a wide range of categories, namely: recommendation (cases \#1, \#2 and \#9), non-compliance (case \#3), question (case \#4), response (case \#5), reminder (case \#6), patient problem message (case \#7), alert note (case \#8), and request for patient clinical information (case \#10). Moreover, the statements show a variety of source and target scenarios.

Table 1. Clinical statements used in our study

\begin{tabular}{|c|c|}
\hline & Clinical Statement \\
\hline $\begin{array}{l}\text { case } \\
\# 1\end{array}$ & $\begin{array}{l}\text { The DSS system provides a recommendation to the patient to measure her Pre-prandial Lunch Blood } \\
\text { Glucose }\end{array}$ \\
\hline case & $\begin{array}{c}\text { The DSS system provides a recommendation to the physician to consider initiating first time insulin } \\
\text { therapy }\end{array}$ \\
\hline case & $\begin{array}{l}\text { The patient did not start the first time insulin therapy by some reason that she specified in the mobile. } \\
\text { The physician includes this fact in her personal health record }\end{array}$ \\
\hline $\begin{array}{c}\text { case } \\
\# 4\end{array}$ & The DSS asks the patient if she is taking insulin (with the message "Are you taking insulin?") \\
\hline
\end{tabular}




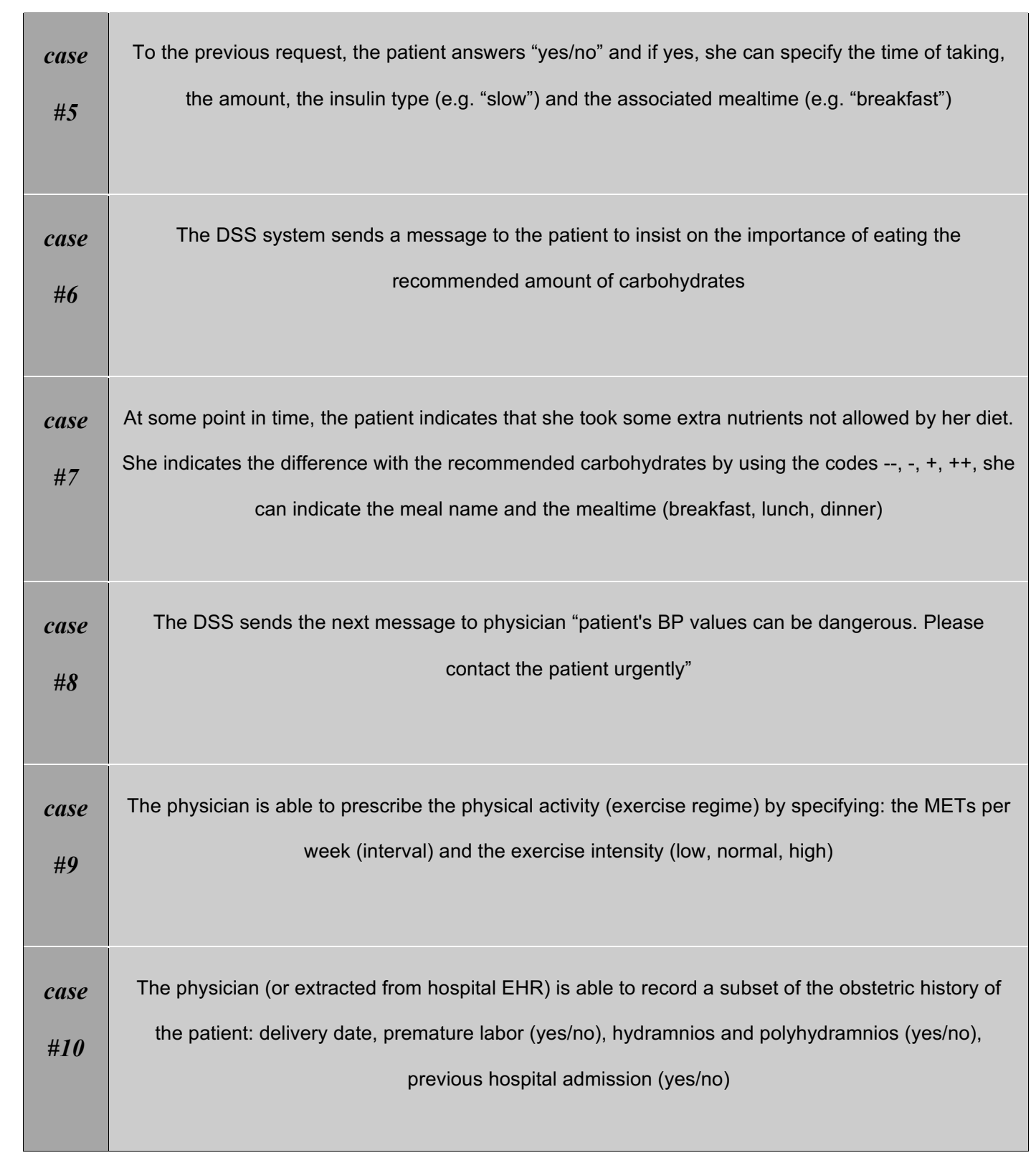

Table 2. Characterization of the clinical statements (cases)

\begin{tabular}{|c|c|c|c|}
\hline & $\begin{array}{c}\text { (General) UMLS } \\
\text { Concept }\end{array}$ & $\begin{array}{c}\text { Source \& } \\
\text { Target }\end{array}$ & Related SNOMED Concepts \\
\hline case & Recommendation & DSS -> & Self-monitoring of blood glucose (procedure), Blood \\
& & PATIENT & $\begin{array}{c}\text { glucose concentration (observable entity), Lunch time } \\
\text { (qualifier value), Before meal (qualifier value), Patient } \\
\end{array}$
\end{tabular}




\begin{tabular}{|c|c|c|c|}
\hline case & Recommendation & $\begin{array}{c}\text { DSS -> } \\
\text { PHYSICIAN }\end{array}$ & $\begin{array}{l}\text { Initiation of first time insulin therapy (regime/therapy), } \\
\qquad \text { Insulin (substance) }\end{array}$ \\
\hline $\begin{array}{c}\text { case } \\
\# 3\end{array}$ & Non-compliance & $\begin{array}{c}\text { PATIENT -> } \\
\text { MOBILE (DSS), } \\
\text { PHYSICIAN -> } \\
\text { EHR }\end{array}$ & $\begin{array}{l}\text { Noncompliance with treatment (finding), Reason for } \\
\text { procedure cancellation (observable entity), Insulin } \\
\text { (substance) }\end{array}$ \\
\hline $\begin{array}{c}\text { case } \\
\# 4\end{array}$ & Question (inquiry) & $\begin{array}{c}\text { DSS -> } \\
\text { PATIENT }\end{array}$ & Direct questioning (procedure), Insulin (substance) \\
\hline $\begin{array}{c}\text { case } \\
\# 5\end{array}$ & Response (statement) & $\begin{array}{l}\text { PATIENT -> } \\
\text { DSS (EHR) }\end{array}$ & $\begin{array}{l}\text { Diabetic on insulin (finding), Diabetes medication } \\
\text { review (procedure), Insulin (substance) }\end{array}$ \\
\hline $\begin{array}{c}\text { case } \\
\# 6\end{array}$ & Reminder & $\begin{array}{c}\text { DSS -> } \\
\text { PATIENT }\end{array}$ & $\begin{array}{l}\text { Reminding (procedure), Diabetes mellitus diet } \\
\text { education (procedure), Patient given advice (situation), } \\
\text { Carbohydrate (substance) }\end{array}$ \\
\hline $\begin{array}{l}\text { case } \\
\# 7\end{array}$ & $\begin{array}{l}\text { Patient problem } \\
\text { message }\end{array}$ & $\begin{array}{l}\text { PATIENT -> } \\
\text { DSS (EHR) }\end{array}$ & $\begin{array}{l}\text { Noncompliance with dietary regimen (finding), Dietary } \\
\text { regime (regime/therapy), Dietary finding (finding) }\end{array}$ \\
\hline $\begin{array}{c}\text { case } \\
\# 8\end{array}$ & Alert note & $\begin{array}{c}\text { DSS -> } \\
\text { PHYSICIAN }\end{array}$ & $\begin{array}{l}\text { Alert received from telehealth monitoring system } \\
\text { (situation), Abnormal blood pressure (finding), } \\
\text { Immediately dangerous to life and health condition } \\
\text { (event) }\end{array}$ \\
\hline $\begin{array}{c}\text { case } \\
\# 9\end{array}$ & Recommendation & $\begin{array}{l}\text { PHYSICIAN -> } \\
\text { PATIENT }\end{array}$ & $\begin{array}{c}\text { Exercise therapy (regime/therapy), Metabolic } \\
\text { equivalent of task (observable entity), With intensity } \\
\text { (attribute) }\end{array}$ \\
\hline
\end{tabular}




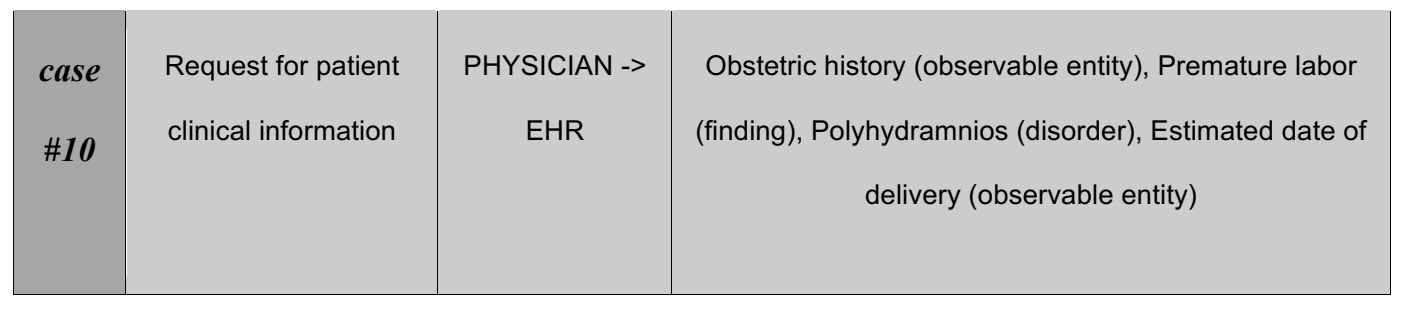

The modelling of instances was done using the vMR XML Schema in the case of vMR, while in the case of openEHR, modelers used either a repository archetype or an archetype specialization that fulfilled the requirements of the statement. Note that the development of such specializations was also part of the modeling task. As illustration, we describe part of the vMR and openEHR solutions developed for statements \#1 and \#10 (figures 1-4, see Appendix B for more details). One vMR solution to statement \#1 (Figure 1) uses an observationProposal with a post-coordinated SNOMED-CT term describing preprandial lunch blood glucose, together with a relatedEntity referring to the patient as the recipient of information. The other vMR representation is roughly the same, except for the use of a procedureProposal. The repository archetype recommendation was used in the case of openEHR (Figure 2) with a participation element specifying that the recipient of the recommendation is the patient (self), plus a textual description in terms of SNOMED-CT terms (including selfmonitoring of blood glucose).

The openEHR solution to statement \#10 (Figure 3) is based on specialization of the pregnancy archetype. Using this archetype as a guide, the information on the pregnancy problems (such as hydramnios/polyhydramnios) has been located under the complications element. This contrasts with the vMR representation of statement \#10 in Figure 4, which comprises a number of elements of type observationResult that are nested beneath an observationResult for the obstetric history. In this way, the modeler has resolved the lack of a specialized structure for this case. 


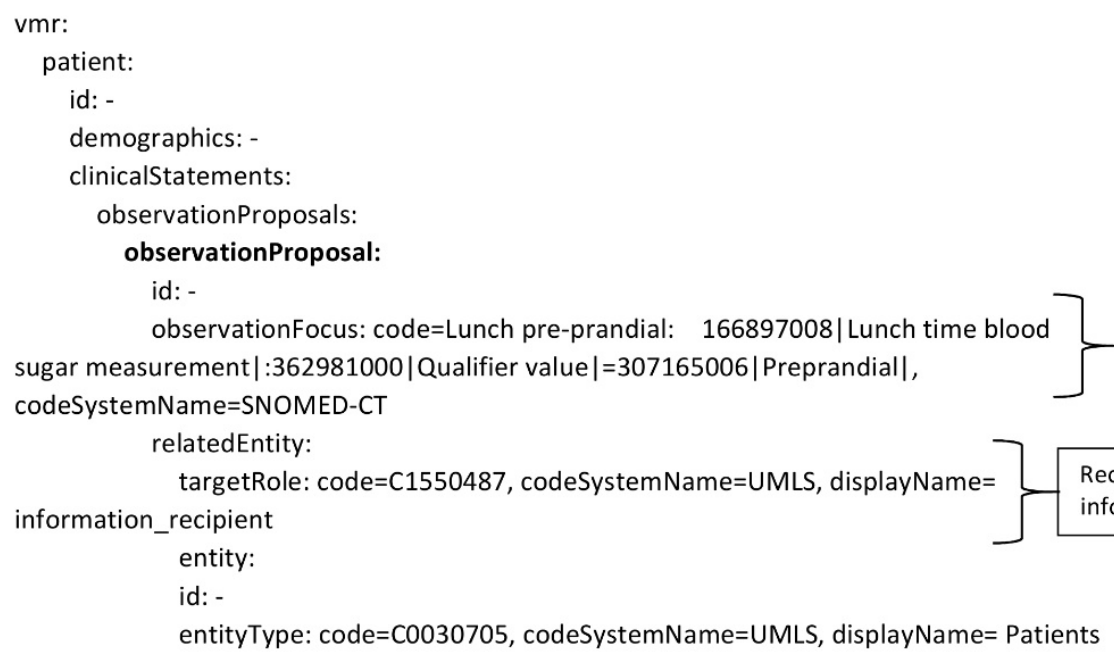

Fig. 1. Schematic representation of one vMR solution to clinical statement \#1.

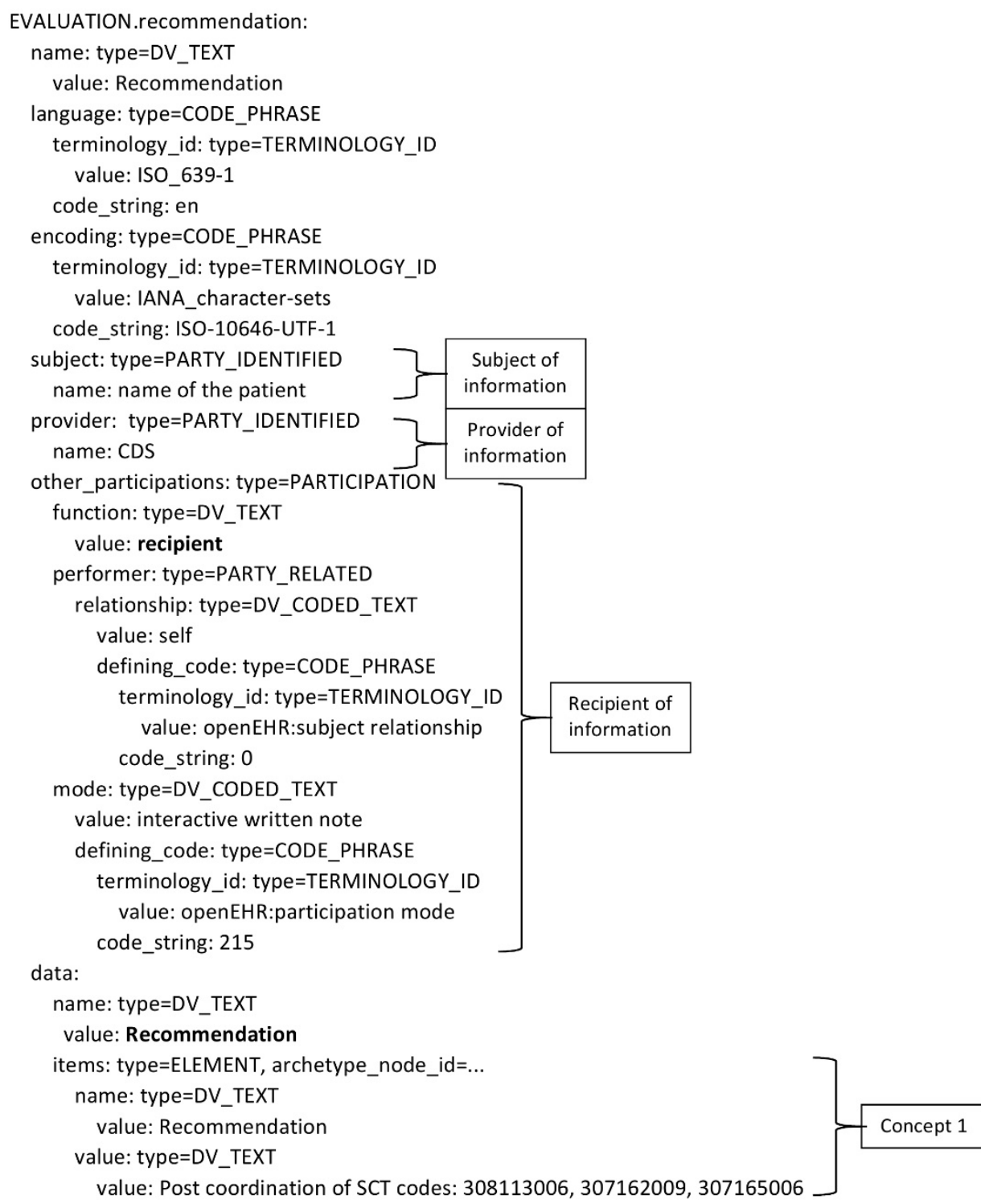

Fig. 2. Schematic representation of the openEHR solution to clinical statement \#1 


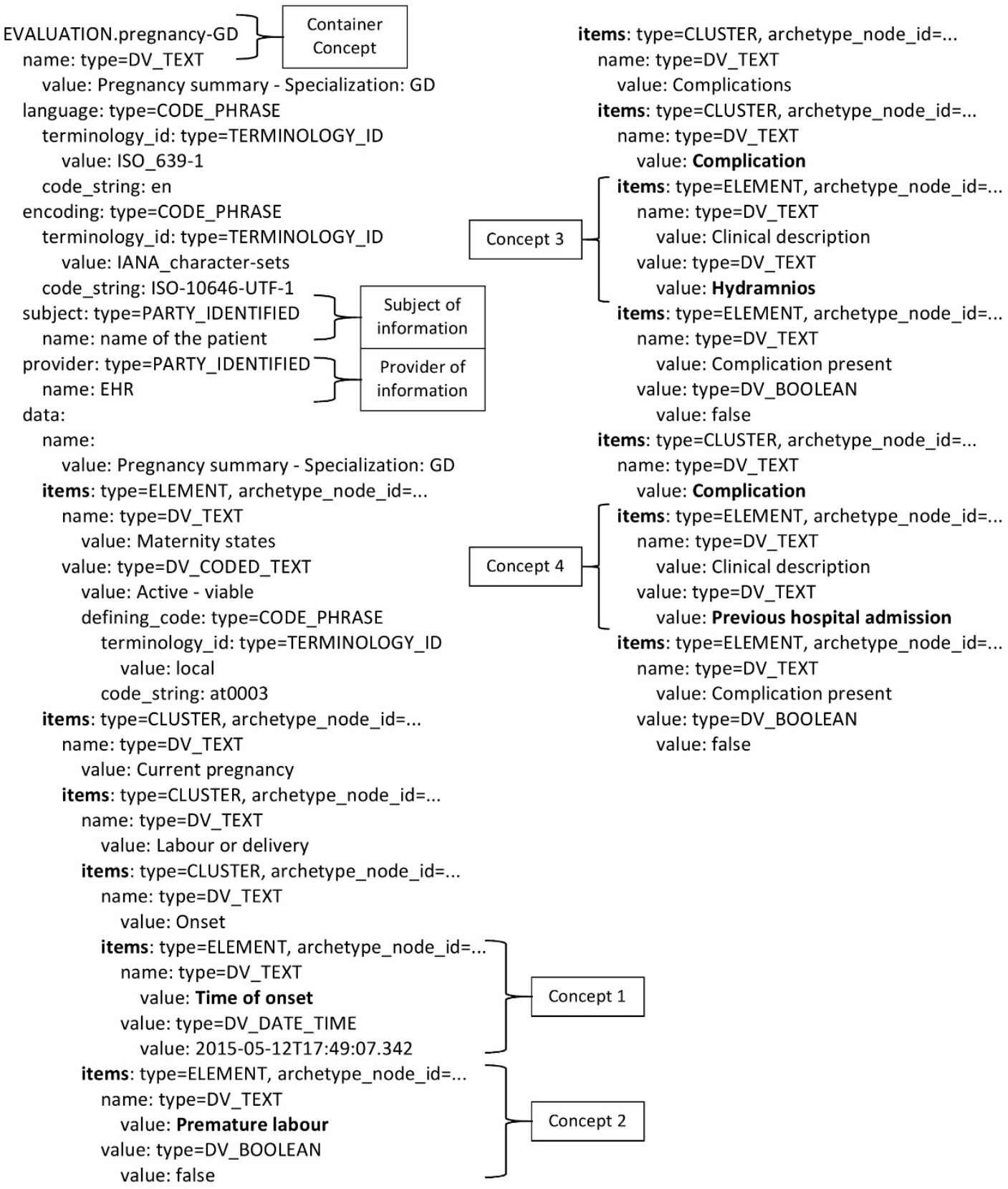

Fig. 3. Schematic representation of the openEHR solution to clinical statement \#10 


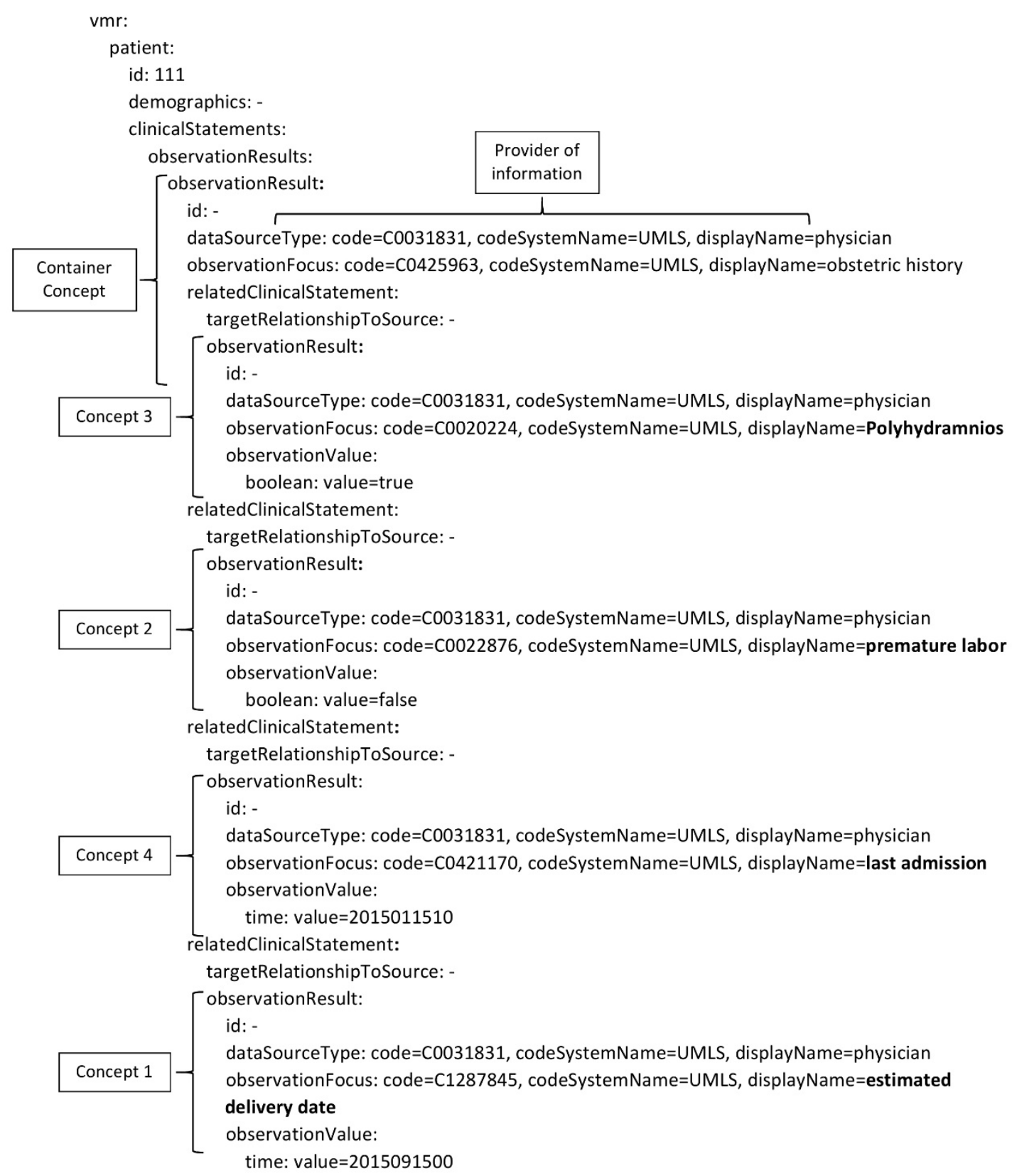

Fig. 4. Schematic representation of one vMR solution to clinical statement \#10

\section{Observation 1: capturing meaning}

In dual model EHR architectures (such as openEHR), three types of entities are used to capture health information meaning: the reference model, archetypes, and terminologies (both the local vocabularies and well-known external terminologies such as SNOMED-CT). In the case of vMR only the reference model and terminologies are used. 
The openEHR reference model, although relatively small compared to other clinical information models such as HL7 CDA, contains formal descriptions of real-world concepts relating to recorded health information, concretely four types of Entries: Observation, Evaluation, Instruction and Action. These entry types are extremely generic and thus archetypes are used to define specific concept models based on each of these types. In archetypes, semantics are captured mostly by a specific and detailed structure with labels, and by the ability to define restrictions (e.g. cardinality, existence or value ranges). Although it is possible to add terminological bindings, the structure is human-understandable by considering the attribute names and labels. Consequently, archetype modelers usually do not specify vocabulary terms. Workflow is captured only for Action entries by means of an attribute (ism_transition) describing the state: in-progress, scheduled, started, etc.

Meaning is captured in vMR by the class type and the use of terminological bindings [5][24]. vMR provides 22 classes that represent real-world concepts relating to recorded health information. They express the type of information and the moment in the workflow (proposal, order, event or undelivered). vMR is more dependent on terminologies than openEHR to capture meaning; this is an important point considering the fact that finding the vocabulary code with the exact meaning is difficult and time consuming. Nevertheless, in both approaches the "boundary problem" is present $[24,25]$, i.e. the way information models and terminologies interplay to unambiguously represent clinical meaning.

\section{Observation 2: structure completeness and flexibility}

Selecting the structure (class or archetype) to use as starting point for the representation of the statement is crucial for both approaches. In the vMR, one of a few classes, namely those representing observation, substance administration and procedure, provided a well-suited solution for most cases. There exist specializations of these classes in terms of the stage of the process, e.g., proposal, order, or result, leading to a range of classes such as SubstanceAdministrationProposal or ObservationResult[26]. However, a wellmatched vMR structure could not be identified for use cases \#9 and \#10, which 
involve the prescription of physical activity and the query of obstetric history parameters, respectively.

In openEHR, the archetypes used mainly belong to the Evaluation category, with varying degrees of specialization, ranging from rather generic archetypes, such as recommendation, to highly specialized and detailed ones like pregnancy. The latter was a particularly good match for case \#10. Nevertheless, in most cases the archetypes were further specialized to fulfill the requirements of use cases by including additional elements and/or constraints. The LinkEHR editor[21] simplified this process.

Finally, there are significant differences between the vMR and openEHR regarding the obligation to include certain structural elements. In openEHR, archetypes serve as an instrument to define a variety of constraints according to the needs of the problem, playing an important role in instance creation and validation. This contrasts with vMR, where the definition of additional constraints is not the prime concern.

\section{Observation 3: encoding variability}

Individual differences in modeling style are a known phenomenon[27]. Both vMR and openEHR archetypes have a highly recursive structure enabling specification of the same elements at different levels. There was little variability between the vMR modelers for clinical statements that matched the regular vMR classes (e.g., SubstanceAdministration or Observation). However, individual differences were noted for more complicated statements. For example, only one of the vMR modelers used the container class shown in Figure 4. In case \#9 one modeler represented the exercise regimen as a ProcedureOrder while the other adapted SubstanceAdministationOrder.

Because the two openEHR experts worked together for the modeling, we could not note individual modeling differences. However, we expect variability in cases where the community-developed archetypes do not exist or have to be extended, but little or no variability for statements that match community-developed archetypes. If a modeler needs to extend an archetype, he can benefit from a detailed archetype developed by the community, which he can follow. This has been done in case \#10 (Figure 3), where a constraint on maternity state was added 
and "hydramnios" was represented as a complication. Individual differences could also be observed when more than one archetype is deemed appropriate. For example, case \#5, could be represented using two different archetypes: checklist (EVALUATION) or medication_order (INSTRUCTION). In both cases, the archetype should be specialized for reporting information specific to insulin intake.

Interestingly, case \#7 demonstrated user variability across the standards used. It required representing patient reporting of consuming an irregular amount $(--,-,+$, ++ ) of carbohydrates during a specific mealtime. As shown in Figure 5, each modeler chose a different representation, using either one or two levels of nesting within a carbohydrate intake item, or one level of nesting within a question item.

(a)

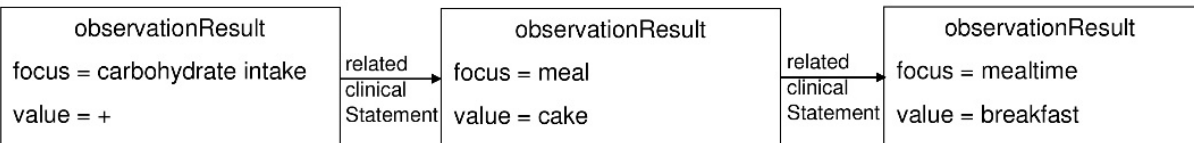

(b)

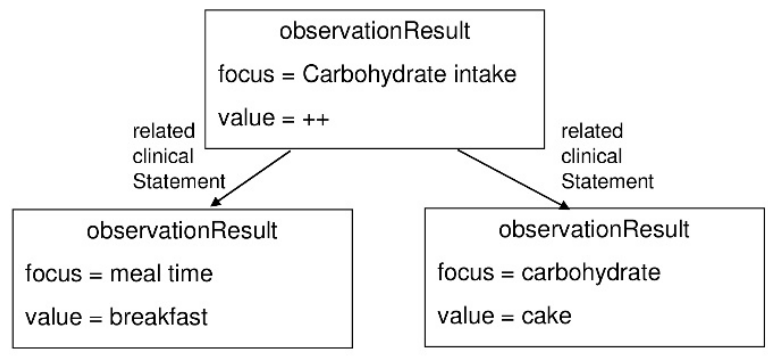

(c)

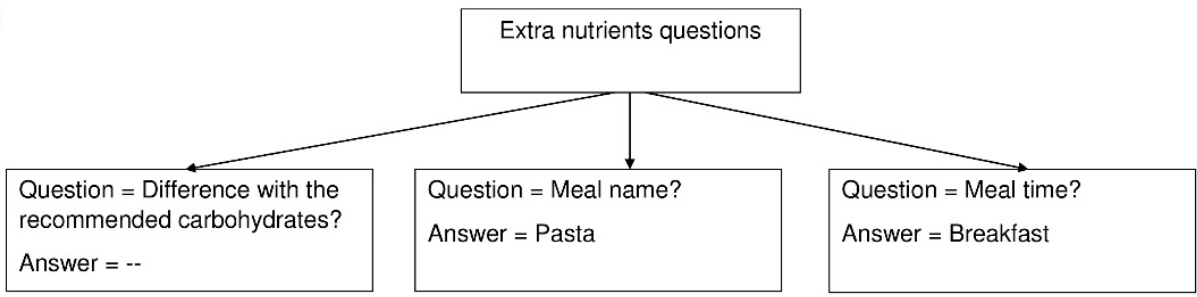

Fig. 5. Schematic representation of the two vMR solutions ((a) and (b)) and the openEHR solution (c) to clinical statement \#7

\section{Observation 4: trade-off between model size and structure completeness and how it potentially affects mapping speed}

In openEHR, it takes longer to select which repository archetype to use and locate the appropriate elements to fill in. Sometimes, specialization of the selected 
archetype was needed (e.g., cases \#4-7), requiring adding new attributes. In our experiments, we observed not only a reduction in the size of vMR instances (between 41-53\%) in comparison to openEHR ones, but also a difference of 4$33 \%$ in size between the two vMR modellings. However, longer times for openEHR may have benefits: more standardization, higher provision of semantics, and possibility for reuse (see Observation 5). In comparison, vMR classes are chosen faster but the adopted solution cannot be easily reused. The inclusion of unavailable attributes in the original class structure is carried out through relatedEntity and relatedClinicalStatement extension mechanisms, which pursue the same goal of specialization but at the instance level. Such generic extension mechanisms increase the variability, e.g. due to the need to express meaning through different vocabulary terms. In the recent vMR Logical Model R2 specification, a user-specified attribute for the ClinicalStatement and Entity classes has been included to support user-defined attribute extensions for clinical concepts, which simplifies the extension mechanism.

There are implementation-related benefits from both approaches. The vMR model has shown to be versatile to implement generic insertion APIs able to deal with the mentioned extensions mechanisms[28]. openEHR has the ability to integrate the full scope of clinical attributes found in EHRs, not just the ones that match the CIG's knowledge. This may be beneficial for secondary use of EHRs (e.g. intelligent data analysis).

\section{Observation 5: reuse capabilities}

A distinctive feature of the openEHR approach is the availability of archetype repositories. Archetypes are community-developed and shareable specifications of clinical information models based on the openEHR reference model. Each archetype represents a clinical concept of a certain significance, and provides a specification that is intended to be as inclusive as possible while allowing specialization. This reflects the fact that openEHR was conceived with the aim of reuse in mind. On the other hand, the vMR is a data model designed to represent clinical data relevant to DSSs, including structural specifications for inputs and outputs and data requirements for specific use scenarios. In a way, there exists also reuse in this case, but it is limited to the relatively simple classes of the vMR. 


\section{Observation 6: recording unconducted activities (e.g., non compliance)}

Case \#3 required reporting of an activity that was supposed to happen (e.g., patient taking medication) but was not conducted and record the reason. The vMR provides support to report unconducted or undelivered activities as well as the reason for not conducting them, which could be useful to understand causes for deviations from recommendations. Since openEHR does not provide a special archetype for this purpose the modeler chose the reason_for_encounter archetype (EVALUATION).

\section{Observation 7: capturing ethical and legal information}

vMR and openEHR have different scopes. vMR is an information model for representing clinical data relevant to DSS. openEHR is a comprehensive EHR architecture and as such it intends to represent any EHR extract. One of the basic EHR architectural requirements is the support for ethical and legal information [3]. Among other information, an EHR architecture shall permit representing the audit trail, authorship, and dates and times of EHR data recording. The ethical and legal information becomes relevant when the DSS output or the information provided by the patient or physician shall be included in the EHR, as in case \#3. openEHR is fully compliant with these requirements[29]. vMR does not provide full support for them and therefore it can be only represented partially. Instead, generic classes combined with terminologies must be used, such as the relatedEntity class for representing authoring information.

\section{Observation 8: ability to specify alerts and reminders}

In both openEHR and vMR two different archetypes/classes are used to represent alerts and reminders. In openEHR the alert archetype (EVALUATION) can be used to represent alerts. On the other hand, although there is no specific archetype for reminders, the health_education archetype (ACTION) can be adopted as solution in the specific context of educational reminders. In both cases, modelers could specialize archetypes to specify a magnitude attribute (urgency, magnitude 
of threat), and in the case of alerts, a possible time frame to respond. On the other hand, vMR R1 has no specific structure for those cases and they both have been modeled as ProcedureEvent instances. In the vMR R2 specification the CommunicationBase class has been added to serve this purpose. It allows a description of the medium, message, reason (e.g., notify, alert, remind), recipient (e.g., person, organization, clinical information system, or device), and sender for this kind of communications.

\section{Discussion and conclusions}

Our collaboration, established among experts on two EHR standards, was very helpful to obtain lessons learned that might be relevant to IT architects who are implementing a DSS which interoperates with the EHR.

We found that the openEHR architecture serves the purpose of data and semantic specification very well, including legal and ethical information, and providing detailed (structure- and terminology-based) semantics. Archetypes have great potential for reuse other than for decision-support, including intelligent data analysis. On the negative side, they require more time, effort, and specialized editing tools (e.g. LinkEHR[21]). Creating instances using the vMR model, on the other hand, requires less time, given that it was specifically designed for the scope of DSS. Its semantics rely to a large extent on vocabulary terms, involving higher variability and scant possibilities for reuse, which also makes difficult the aim of standardization itself. As advantage, it serves very well for fast prototyping of pilot projects, as was already demonstrated in MobiGuide [28]. In this sense, variability could be limited by developing agreed-upon templates. The results of our study could serve as useful input for the team in HL7 that started defining such templates [30], benefiting from our experience with the CKM archetype repository as well.

This study has some limitations. The exploration is limited to a small set of clinical statements, so there are certainly issues that could have gone unnoticed after our analysis. Furthermore, modelers with expertise in one standard were dealing with exactly that standard, which might imply different considerations to those that unexperienced users could have found. 


\section{Acknowledgements}

This study was partially funded by the European Commission 7th Framework

Program, grant \#287811. It has also been supported by the Spanish Ministry of Economy and Competitiveness and the EU FEDER programme through project TIN2014-53749-C2-1-R and grant PTQ-12-05620.

\section{References}

1. Peleg M (2013) Computer-interpretable Clinical Guidelines: a Methodological Review. J Biomed Inform 46:744-763

2. Peleg M, Shahar Y, Quaglini S (2014) Making healthcare more accessible, better, faster, and cheaper: the MobiGuide Project. Eur J e-Practice 20:5-20

3. ISO (2011) ISO 18308:2011. Health Informatics-Requirements for an Electronic Health Record Architecture. http://www.iso.org/iso/catalogue_detail?csnumber=52823. Accessed 29 Dec 2015

4. Lenzerini M (2002) Data integration: A theoretical perspective. In: Popa L (ed) Proc. 21st ACM SIGMOD-SIGACT-SIGART Symp. Princ. database Syst. pp 233-246

5. Peleg M, Keren S, Denekamp Y (2008) Mapping computerized clinical guidelines to electronic medical records: knowledge-data ontological mapper (KDOM). J Biomed Inform 41:180-201

6. Marcos M, Maldonado JA, Martínez-Salvador B, Boscá D, Robles M (2013) Interoperability of clinical decision-support systems and electronic health records using archetypes: a case study in clinical trial eligibility. J Biomed Inform 46:676-689

7. Friedman M, Levy AY, Millstein TD, others (1999) Navigational plans for data integration. In: Hendler J, Subramanian D (eds) Proc. 16th Natl. Conf. Artif. Intell. AAAI Press, Orlando, Florida, pp 67-73

8. Parker CG, Ms SWT, Huff SM (2004) Detailed Clinical Models for Sharable, Executable Guidelines. In: Fieschi M, Coiera E, Li Y-CJ (eds) Proc. 11th World Congr. Med. Informatics. IOS Press, San Francisco (USA), pp 145-148

9. Tao C, Jiang G, Oniki TA, Freimuth RR, Zhu Q, Sharma D, Pathak J, Huff SM, Chute CG (2013) A semantic-web oriented representation of the clinical element model for secondary use of electronic health records data. J Am Med Informatics Assoc 20:554-562

10. German E, Leibowitz A, Shahar Y (2009) An architecture for linking medical decisionsupport applications to clinical databases and its evaluation. J Biomed Inf 42:203-218

11. Cho I, Kim J, Kim JH, Kim HY, Kim Y (2010) Design and implementation of a standardsbased interoperable clinical decision support architecture in the context of the Korean 
EHR. Int J Med Inform 79:611-622

12. Tu SW, Campbell JR, Glasgow J, et al (2007) The SAGE Guideline Model: achievements and overview. J Am Med Informatics Assoc 14:589-598

13. Health Level Seven (2014) HL7 Version 3 Standard: Clinical Decision Support; Virtual Medical Record (vMR) Logical Model, Release 2.

http://www.hl7.org/implement/standards/product_brief.cfm?product_id=338. Accessed 14 Jan 2016

14. Marcos M, Maldonado J, Martínez-Salvador B, Moner D, Boscá D, Robles M (2011) An archetype-based solution for the interoperability of computerised guidelines and electronic health records. In: Peleg M, Lavrač N, Combi C (eds) Artif. Intell. Med. Springer, Bled, Slovenia, pp 276-285

15. González-Ferrer A, Peleg M (2015) Understanding requirements of clinical data standards for developing interoperable knowledge-based DSS: A case study. Comput Stand Interfaces 42:125-136

16. González-Ferrer A, Peleg M, Verhees B, Verlinden JM, Marcos C (2013) Data Integration for Clinical Decision Support Based on openEHR Archetypes and HL7 Virtual Medical Record. In: Lenz R, Miksch S, Peleg M, Reichert M, Riaño D, ten Teije A (eds) Proc. BPM Jt. Work. ProHealth12/KR4HC12 (LNAI 7738). Springer, Tallinn, Estonia, pp 7184

17. January CT, Wann LS, Alpert JS, et al (2014) 2014 AHA/ACC/HRS guideline for the management of patients with atrial fibrillation: a report of the American College of Cardiology/American Heart Association Task Force on Practice Guidelines and the Heart Rhythm Society. J Am Coll Cardiol 64:e1-e76

18. Health NCC for W and C (2008) Guidelines: management of diabetes from preconception to the postnatal period: summary of NICE guidance. BMJ Br Med J 336:714

19. Lindberg C (1990) The Unified Medical Language System (UMLS) of the National Library of Medicine. J Am Med Rec Assoc 61:40-42

20. NLM (2009) SNOMED Clinical Terms (SNOMED CT). http://www.ihtsdo.org/snomedct. Accessed 14 Jan 2016

21. Maldonado JA, Moner D, Boscá D, Fernández-Breis JT, Angulo C, Robles M (2009) LinkEHR-Ed: A multi-reference model archetype editor based on formal semantics. Int J Med Inform 78:559-570

22. von Alan RH, March ST, Park J, Ram S (2004) Design science in information systems research. MIS Q 28:75-105

23. Fink A, Kosecoff J, Chassin M, Brook RH (1984) Consensus methods: characteristics and guidelines for use. Am J Public Health 74:979-983

24. Markwell D, Sato L, Cheetham E (2008) Representing Clinical Information using $\underline{\text { SNOMED Clinical Terms with Different Structural Information Models. In: Cornet R, }}$ Spackman K (eds) Proc. Third Int. Conf. Knowl. Represent. Med. CEUR, Phoenix, Arizona, pp 72-79 
25. Rector AL, Qamar R, Marley T (2009) Binding ontologies and coding systems to electronic health records and messages. Appl Ontol 4:51-69

26. González-Ferrer A, Peleg M, Parimbelli E, Shalom E, Lagunar CM, Klebanov G, Martínez-Sarriegui I, Fung NLS, Broens T (2014) Use of the Virtual Medical Record Data Model for Communication among Components of a Distributed Decision-Support System. In: Proc. 2nd IEEE Biomed. Heal. Informatics Int. Conf. IEEE Computer Society, Valencia, Spain, pp 526-530

27. Patel VL, Branch T, Wang E, Peleg M, Boxwala A, others (2002) Analysis of the process of encoding guidelines: a comparison of GLIF2 and GLIF3. Methods Inf Med 41:105-113

28. Marcos C, González-Ferrer A, Peleg M, Cavero C (2015) Solving the interoperability challenge of a distributed complex patient guidance system: A data integrator based on HL7's Virtual Medical Record standard. J Am Med Assoc 22:587-599

29. Beale T (2006) ISO 18308 Conformance Statement release 1.0.1.

30. Health Level Seven (2015) HL7 Version 3 Standard: Clinical Decision Support; Virtual Medical Record (vMR) Templates, Release 1.

http://www.hl7.org/implement/standards/product_brief.cfm?product_id=339. Accessed 14 Jan 2016 